\title{
D-Mason: A Short Tutorial
}

\author{
Vittorio Scarano $^{1}$, Gennaro Cordasco ${ }^{2}$, \\ Rosario De Chiara $^{3}$, and Luca Vicidomini ${ }^{1}$ \\ 1 ISISLab - Dipartimento di Informatica \\ Università di Salerno, Italy \\ vitsca@ia.unisa.it, lvicidomini@unisa.it \\ 2 Dipartimento di Psicologia \\ Seconda Università degli Studi di Napoli, Italy \\ gennaro.cordasco@unina2.it \\ ${ }^{3}$ Centro Ricerca e Sviluppo \\ Poste Italiane, Italy \\ dechia24@posteitaliane.it
}

\begin{abstract}
D-MASON framework is a parallel version of the MASON library for writing and running Agent-based simulations. We briefly present MASON architecture, functionalities and some programming examples. Full documentation, additional tutorials and other material can be found at www. dmason. org where the framework can be downloaded.
\end{abstract}

\section{D-Mason: Motivations and Design}

Agent-Based simulation Models (ABMs) are an increasingly popular tool for research and management in many, different and diverse fields. In executing such simulations the "speed" is one of the most general and important issues and the traditional answer to this issue is to invest resources in deploying a dedicated installation of dedicated computers, with highly specialized parallel applications, devoted to the purpose of achieving extreme computational performances.

Here we present a framework, D-MASON [3], that is a distributed version of MASON, a well-known and popular library for writing and running Agent-based simulations. D-MASON introduces the parallelization at framework level so that scientists that use the framework (domain expert but with limited knowledge of distributed programming) can be only minimally aware of such distribution.

\subsection{MASON}

Before presenting the architecture of D-MASON in the next subsections we will, first, introduce MASON toolkit [5]6], a discrete-event simulation core and visualization library written in Java, designed to be used for a wide range of ABMs. The toolkit is written, using the standard Model-View-Controller (MVC) paradigm, in three layers: the simulation layer, the visualization layer and the utility layer. The simulation layer is the core of MASON and is mainly represented by an event scheduler and a variety of fields which hold agents into a given simulation space. MASON is mainly based on

D. an Mey et al. (Eds.): Euro-Par 2013 Workshops, LNCS 8374, pp. 490-500, 2014.

(c) Springer-Verlag Berlin Heidelberg 2014 


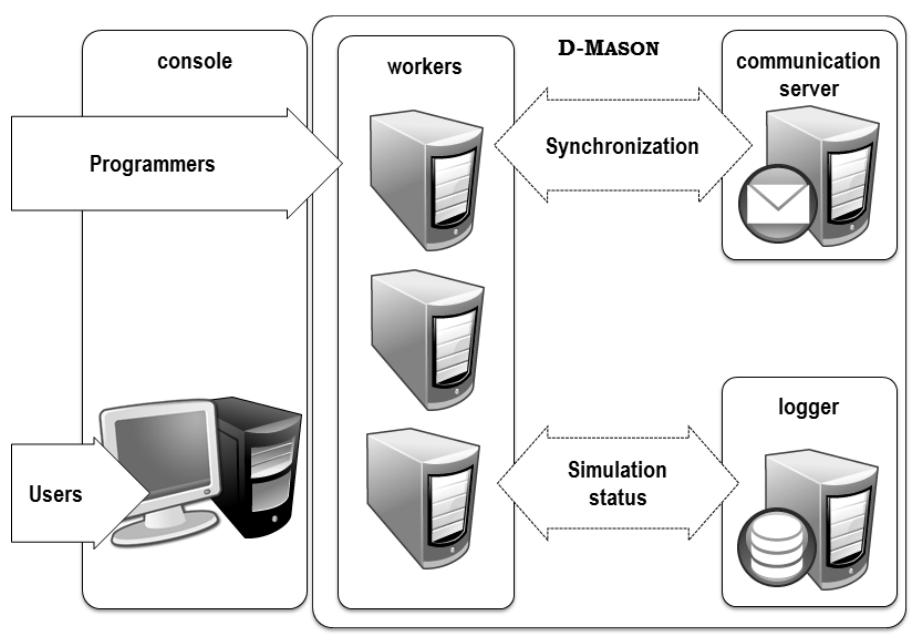

Fig. 1. D-Mason scheme

step-able agent: a computational entity which may be scheduled to perform some action (step), and which can interact (communicate) with other agents. The visualization layer permits both visualization and manipulation of the model. The simulation layer is independent from the visualization layer, which allows us to treat the model as a self-contained entity.

MASON was written with the aim of creating a flexible and efficient ABM framework which assures the complete reproducibility of results across different hardware. This reproducibility feature is considered as a priority for long simulations: for instance it is possible stop a simulation and move it a faster computer once it became available.

\subsection{D-MASON}

D-MASON is based on a master/workers paradigm (see Figure 1): the master console assigns a portion of the whole computation (i.e., a set of agents) to each worker. Then for each simulation step, each worker simulates the agents assigned and sends back the result of its computation to each interested worker.

\section{D-Mason Features}

Field partitioning. By noticing that most ABMs are inspired by natural models, where agents limited visibility allow to bound the range of interaction to a fixed range named agent's Area of Interest (AOI), several space partitioning approaches have been proposed [478] in order to reduce the communication overhead. In D-MASON, the space to be simulated (D-MASON field) is partitioned into regions (see Figure 2 left). Each region, together with the agents contained in it, is assigned to a worker. Since the AOI of an agent is small compared with the size of a region, the communication is limited to local messages (messages between workers, managing neighboring spaces, etc.). The 

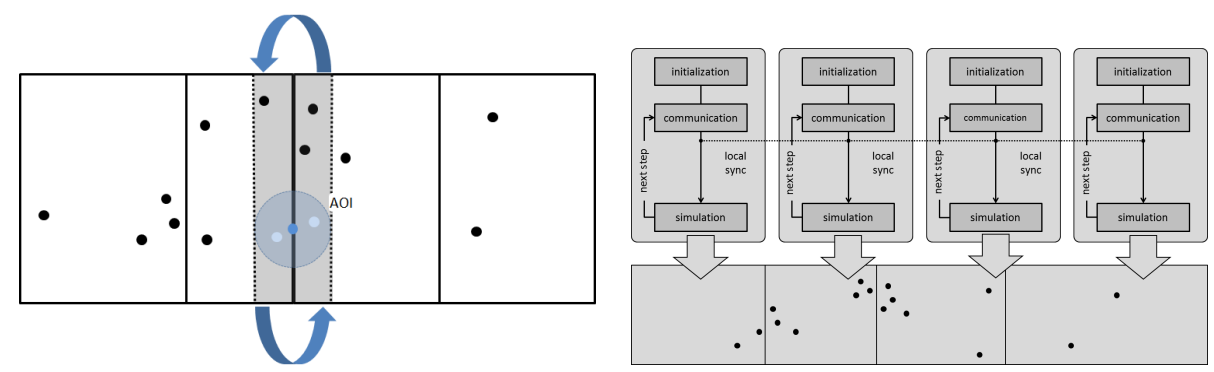

Fig. 2. Field partitioning and Synchronization

problem with this approach is that agents can migrate between regions and consequently the association between workers and agents changes during the simulation. Moreover, load balancing is not guaranteed and need to be addressed by the application.

Synchronization. We view ABMs as step-wise computations; i.e., agents behavior is computed in successive steps named simulation step. In order to guarantee the consistency of parallel implementation with respect to the sequential one, each worker needs to collect information about the neighboring regions. Each simulation step is formed by two phases: communication/synchronization and simulation. First of all, the worker $w$ sends to its neighbors (i.e., the workers responsible for its neighbor regions) the information about:

- the agents that are migrating to them;

- the agents that may fall into the AOI of the agents in neighborhood of $w$.

This information exchange is locally synchronized in order to let the simulation run consistently (see Figure 2 right). We use a standard approach to achieve a consistent local synchronization of the distributed simulations: each step is associated with a fixed state of the simulation. Regions are simulated step by step. Since the step $i$ of region $r$ is computed by using the states $i-1$ of $r$ 's neighborhood, the step $i$ of a region cannot be executed until the states $i-1$ of its neighborhood have been computed and delivered. In other words, each region is synchronized with its neighborhood before each simulation phase.

Communication. D-MASON uses a well-known mechanism, based on the publishsubscribe design pattern, to propagate agents state information: a multicast channel is assigned to each region; workers, then, simply subscribe to the channels associated with the regions which overlap with their AOI to receive relevant message updates. The current version of D-MASON uses Java Message Service (JMS) for communication between workers. We use a communication server, running Apache ActiveMQ Server, that acts as a JMS provider (i.e., it allows to generate and manage multicast channels and route messages accordingly).

Reproducibility. In order to guarantee an easy parallelization and to assure the reproducibility of results, paramount objective of the research areas interested in the ABMs, 
it is important to design the simulation is such a way that agents evolves simultaneously. Using this approach the simulation become embarrassingly parallelizable (there are no dependencies between agents' state), each simulation step can be executed in parallel overall the agents. Moreover, the order in which agents are scheduled does not affect the reproducibility of results.

Load Balancing. D-MASON uses a simple but efficient technique to cope with heterogeneity. The idea is to clone the software run by high capable workers so that they could serve as multiple workers; i.e., a worker that is $x$ times more powerful than other workers could execute $x$ virtual workers (that is by simulating, concurrently, several regions).

\section{D-MASON Functionalities}

D-MASON architecture is divided into four functional blocks: Management, Workers, Communication and Services (see Figure 1). The Management layer provides a master application which will be used for coordinating the workers, handle the bootstrap and running the simulation. The master is responsible for partitioning the field into regions and assigning them to workers. Currently in D-MASON there are two types of field partitioning: horizontal, where the division is done by splitting the field along one axis and square, where the division is done by using a grid. When all the parameter are set it is possible to start and interact with the entire distributed simulation (e.g. play, pause, stop). The workers are in charge of: simulating the agents that belong to the assigned regions; handling the migration of agents; managing the synchronization between neighboring regions. Workers communicate by using the communication layer based on a publish-subscribe mechanism. Services, logging and visualization (both are optional), interact with the communication server to obtain data for the simulation.

\section{Programming with D-MASON}

Here is a brief description of the steps one should take to make existing MASON applications run in the D-MASON.

First of all, small changes to the application source code have to be done: the new D-MASON application should extend Distributedstate instead of Simstate. The field to be distributed should be the distributed version of the original one. For instance, if the application is based on SparseGrid2D its distributed version should use DSparseGrid2D. Several additional parameter should be added to the application: type of partitioning (horizontal or square), AOI range and number of regions. Moreover some additional parameters need to be defined to setup the communication layer (for instance, in the current version we are using Apache ActiveMQ, and in this case, the IP address and Port number of the Communication Server should be provided).

In order to instantiate the distributed field, D-MASON provides a specific Factory. For instance, DSparseGrid2DFactory . createDSparseGrid2D ( . . .) is used to generate a DSparseGrid2D field. This field is the core of the distributed simulation and allows both the simulation and synchronization phases. Each 
region of the distributed field owns a pseudo-random number generator (i.e., a MersenneTwisterFast object).

Another important change that should be made on the distributed application is to change the method used to add agents to the schedule. In MASON applications, agents are usually added to the schedule using the method scheduleRepeating ( ) . In DMASON all the agents are scheduled after each synchronization phase using the method scheduleOnce ( ). Hence scheduleRepeating ( ) should never be used in order to avoid scheduling migrated agents.

The method addToField () of the abstract class Distributedstate should be implemented, according to the behavior of the application.

Each agent, used by the application, should extends the abstract class RemoteAgent that implements Steppable. The method step ( ) that defines agent action must take into account that the simulation should evolve simultaneously (see Section 1.2). Hence each agent computes its new state and store it in a temporary buffer. When all the states are computed, all the states are updated atomically. The new location of the agent is set through the method setDistributedobjectLocation ( ) which implements the whole logic of agents movements (i.e., it checks whether the agent migrate or not, or whether the agent should be sent to a neighbor because it may fall into one of its agents' AOI.)

We have also implemented three distributed ABMs which are based on existing MASON applications: DParticle, DFlockers and DAntsForage which use DSparseGrid2D, DContinuous2D, DDoubleGrid2D, DIntGrid2D as field.

In the following we intend to provide a clear view of what means for the average MASON user to migrate his/her simulation to D-MASON in order to fully exploit the possibilities of a distributed simulation.

To better clarify we will migrate the Particle example which is readily available among the packages of MASON.

\section{From Particles to DParticles}

the package Particles is composed by three classes, as it can be found in the original MASON distribution: Particle, it implements the agent that will be simulated by the application; Particles, it represents the simulation environment: it allows to run the simulation from the command line without using a GUI; ParticlesWithUI, it allows to run simulations with a GUI.

Similarly, in D-MASON, the package DParticles containing the following classes: RemoteParticle, an abstract class, implementing RemoteAgent and containing the remote ID of the agent in the field and its position; DParticle, it extends RemoteParticle and implements the distributed agent that will be simulated by the application; DParticles, it represents the distributed simulation environment: it allows to run the simulation from the command line without using a GUI; DParticlesWithUI, it allows to run simulations with a GUI that is aware of the distributed environment.

RemoteAgent is parameterized with an Int2D object-type because, in this simulation, the field has this specific type to indicate locations, and allows programmers 
to set, for each agent, an unique identifier and a field position. A Particle simply contains two integer parameters, xdir and ydir, for setting the initial direction that the particle will move along.

DParticle has two constructors: the first is empty and it has been introduced for a future implementation of the method clone (), and the second one has as parameter, a subclass of the abstract class Distributedstate.

\section{Listing 1.1. Class Particle}

$\cdots$

public Particle(int xdir, int ydir) \{

public boolean randomize = false;

this.xdir = xdir;

this.ydir = ydir;

\}

$\cdots$

\section{Listing 1.2. Class DParticle}

public class DParticle extends RemoteParticle<Int2D>

\{

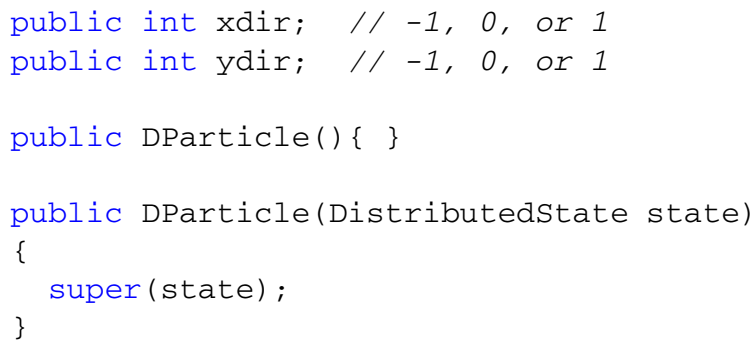

Particles extends the Simstate class while DParticles extends Distributedstate, parameterized with Int2D object-type.

DParticles contains three other variables indicating, respectively, width and height of the field and the way of partitioning the field (that can be one or two dimensions). Particles has just one constructor that has as parameter the random generator seed while DParticles constructor has as input an objects array, containing several parameters specific for the distributed simulation (e.g. network address, port, etc ...).

In Particles there are two fields, the first containing the agents, the second one containing the trails. The creation of the fields and the placement of the agents in them are carried out by a simple loop that instantiates new particles with a random position and direction and place them in the proper field.

In order to add particles to the schedule, it is possible to use scheduleRepeating(), that allows to schedule agents repeatedly, and to add particles to the field there is setobjectLocation( ). In DParticles there is the method createDSparseGrid2D of the class DSparseGrid2DFactory for creating a new distributed field. Note that it is necessary to use a factory to choose 
the kind of field partition. The agent initial position is computed by the method setAvailableRandomLocation() and to add particles in the schedule it is necessary to use the method scheduleOnce (), because in the next step a certain agent could not stay in the same part of the field, so using scheduleRepeating ( ) will not delete the particle from the schedule. Finally there are other three new methods: a getter method for returning the subclass of the Distributedstate, a method for adding an agent with a given position in the field, a method for attaching a portrayal to an agent.

\section{Listing 1.3. Class Particles}

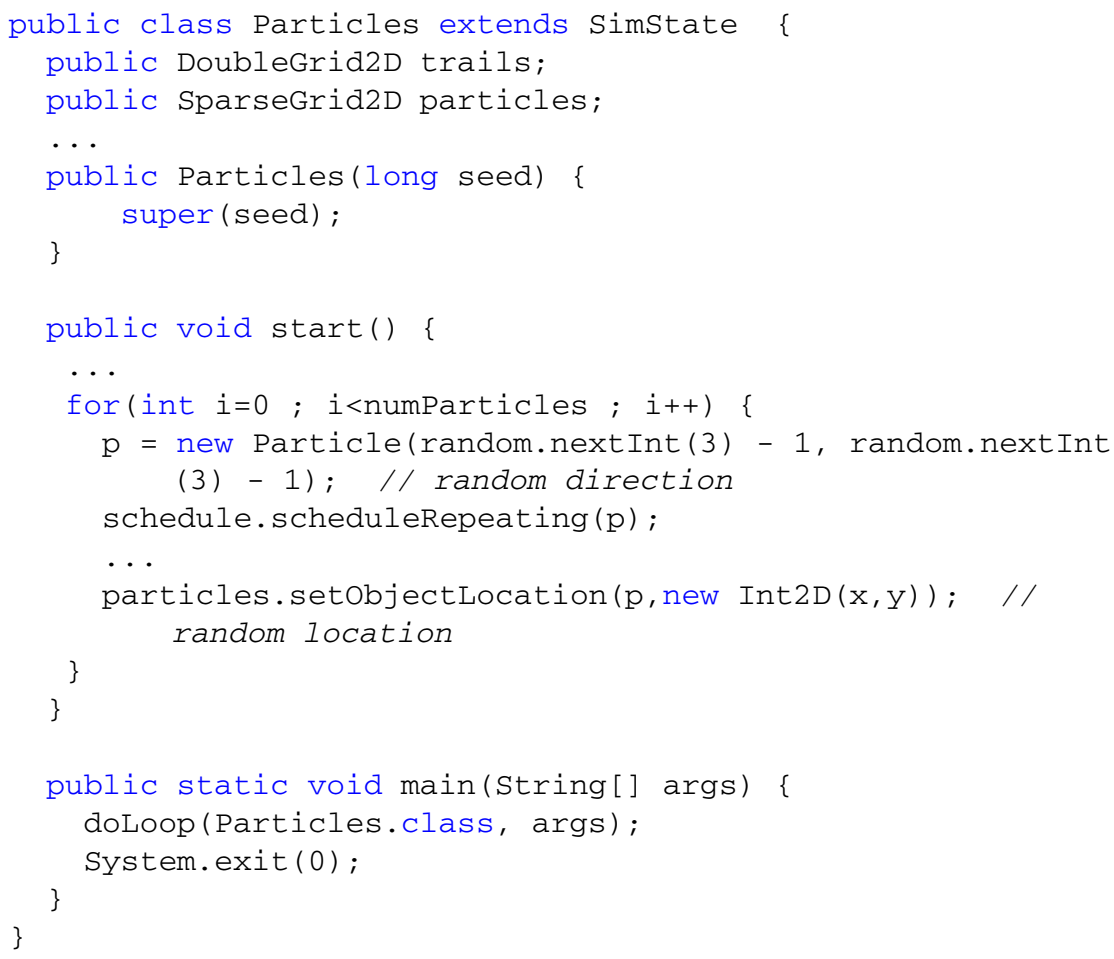

\section{Listing 1.4. Class DParticles}

public class DParticles extends Distributedstate<Int2D $>$ \{

static final long serialVersionUID = 9115981605874680023L;

private static boolean istoroidal = false;

public DSparseGrid2D particles;

public DDoubleGrid2D trails;

public int gridWidth;

public int gridHeight;

public int MODE;

public DParticles(Object[] params) \{

super ( (Integer) params [2] , (Integer) params [3],...); 


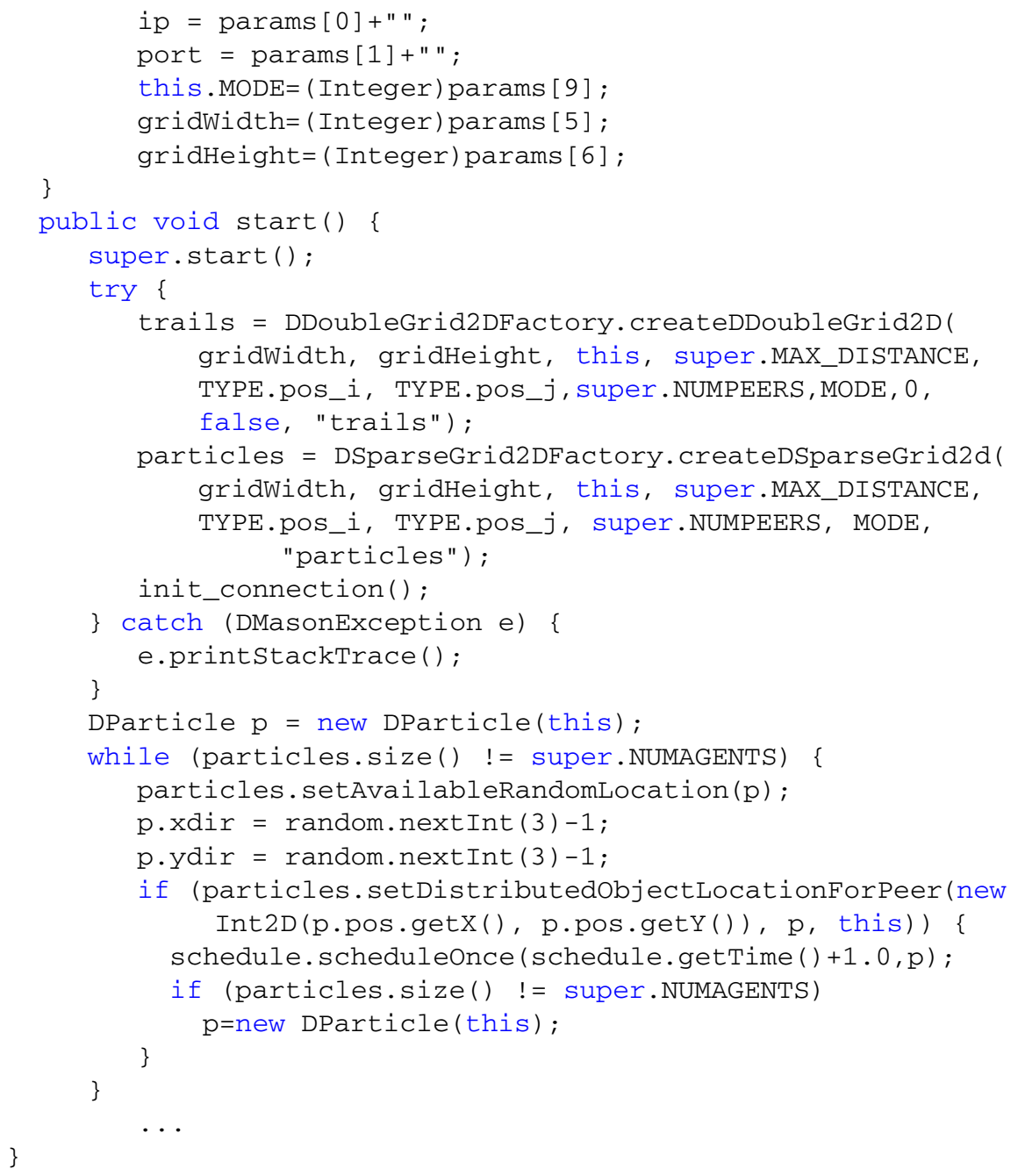

There are few differences between original ParticlesWithUI and the its distributed version, DParticlesWithUI. They both extend the class GUIState, responsible of instantiating all graphics elements; DParticlesWithUI has a constructor which allow to initialize all the parameter fo DParticles. The region identifier is stored in the variable name and is used to show which region is currently visualized.

\section{Listing 1.5. Class ParticlesWithUI}

public class ParticlesWithUI extends GUIState \{

...

public static void main(String[] args) \{

ParticlesWithUI $t=$ new ParticlesWithUI();

t.createcontroller(); 


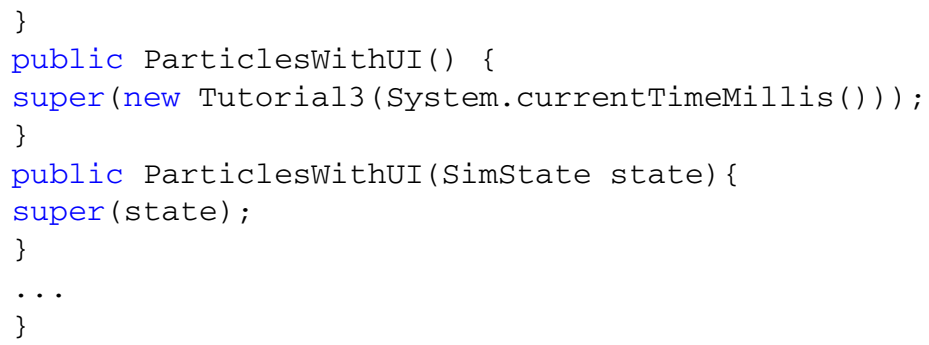

\section{Listing 1.6. Class DParticleswithuI}

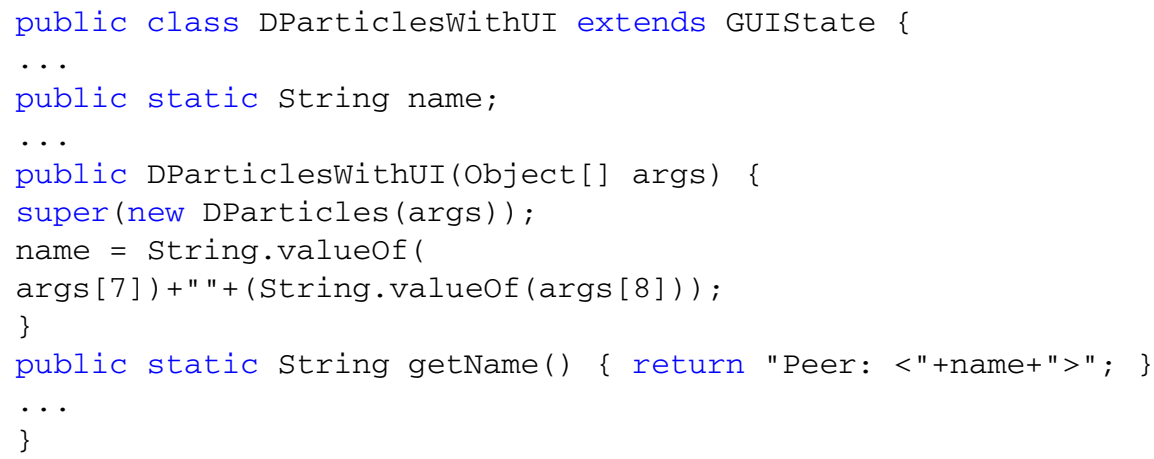

\section{The User'S View: How to Exploit Distributed Simulations}

In the process of designing an $\mathrm{ABM}$ it greatly helps to use facilities that allow to visualize what is happening and to collect the low level data (e.g. position of agents, values of associated properties etc...) for further analysis. In MASON each simulation is developed together with the visualization, called portrayal, that lets the user to understand what is going on. In D-MASON this mechanism is extended to the workers and each worker is able to correctly visualize the region it is in charge of simulating. Of course this is not a suitable solution to be used in any real setting: the scientist should physically move from worker to worker to check what is happening. Furthermore the visualization running on each worker can be considered as a wast of computing power that could be better employed to carry out the simulation.

For the reasons listed above, together with D-MASON, we have implemented a logging facility that is also leveraged to provide a centralized visualization mechanism. The logger (see Figure 11) is in charge of memorize, on each step, the status of the simulation: at the end of a step, each worker sends to it the status of the region it is in charge of. The logger is also used to provide a centralized visualization tool that allows the user to become aware of what is happening. It is possible to use the data collected by the logger to obtain two different centralized visualization (a) a global one which provides a simplified view of the whole field and this allows to just have a simplified 

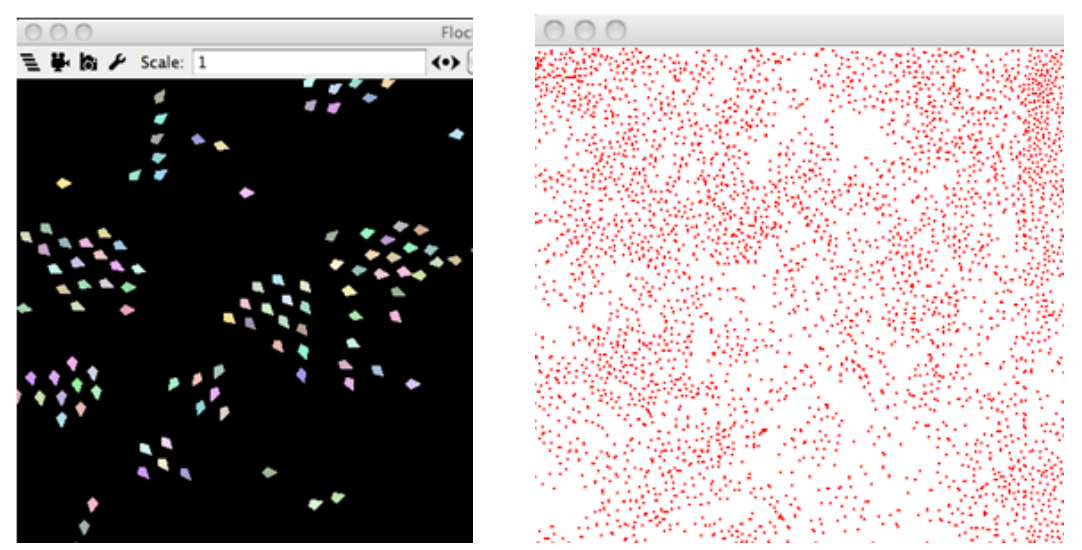

Fig. 3. The two rendering modes: (left) visualization from the portrayal associated to the flocker demo; (right) global visualization showing just the position of the agents

and efficient representation of what is happening (see Figure 3, left); (b) the visualization offered by the portrayal for a specific region of the field (see Figure 3 , right). The reason to offer the two modes is that while the (b) is the mode that offers everything is needed to understand the semantic of the simulation, the mode (a) is lighter from the computational point of view so it is more responsive for a rapid look at the simulation. The two visualization modes are implemented within the console application the user can always switch between them.

\section{Discussion and Conclusion}

This paper reports on an currently undergoing project, D-MASON, that has been developed with the purpose of speeding up the performances of MASON, a very well known and quite widespread framework for ABMs. Several improvements, a better load balancing [1] and a computational steering facility [2], have been introduced to the framework. Further work is already planned to increase the functionality of the system. Finally, the project is available at $w w w$. dmason. org under a Free and Open Software license.

\section{References}

1. Carillo, M., Cordasco, G., De Chiara, R., Raia, F., Scarano, V., Serrapica, F.: Enhancing the Performances of D-MASON - A Motivating Example. In: SIMULTECH, pp. 137-143. SciTePress (2012)

2. Cordasco, G., De Chiara, R., Raia, F., Scarano, V., Spagnuolo, C., Vicidomini, L.: Designing computational steering facilities for distributed agent based simulations. In: Proc. of ACM SIGSIM Conference on Principles of Advanced Discrete Simulation (PADS 2013) (2013) 
3. Cordasco, G., De Chiara, R., Mancuso, A., Mazzeo, D., Scarano, V., Spagnuolo, C.: Bringing together efficiency and effectiveness in distributed simulations: the experience with DMASON. In: SIMULATION: Transactions of The Society for Modeling and Simulation International (2013)

4. Cosenza, B., Cordasco, G., De Chiara, R., Scarano, V.: Distributed load balancing for parallel agent-based simulations. In: Proc. of the 19th Euromicro International Conference on Parallel, Distributed and Network-Based Computing, PDP 2011 (2011)

5. Luke, S., Cioffi-Revilla, C., Panait, L., Sullivan, K.: MASON: A new multi-agent simulation toolkit. In: Proceedings of the 2004 SwarmFest Workshop, Ann Arbor, Michigan, USA, May 9-11 (2004)

6. Luke, S., Cioffi-Revilla, C., Panait, L., Sullivan, K., Balan, G.: MASON: A Multiagent Simulation Environment. Simulation 81(7), 517-527 (2005)

7. Zhang, Y., Mueller, F., Cui, X., Potok, T.: Large-Scale Multi-Dimensional Document Clustering on GPU Clusters. In: IEEE International Parallel and Distributed Processing Symposium (2010)

8. Zhou, B., Zhou, S.: Parallel simulation of group behaviors. In: WSC 2004: Proceedings of the 36th Conference on Winter Simulation, pp. 364-370. Winter Simulation Conference (2004) 